\title{
La méthode de la pensée à voix haute pour analyser les difficultés en lecture des élèves de 14 à 17 ans
}

High School Students Reading's Difficulties Analyzed with Think Aloud Protocols

\author{
Érick Falardeau, Cindy Pelletier et Daisy Pelletier
}

\section{(2) OpenEdition}

\section{Journals}

\section{Édition électronique}

URL : https://journals.openedition.org/educationdidactique/2022

DOI : $10.4000 /$ educationdidactique.2022

ISSN : 2111-4838

\section{Éditeur}

Presses universitaires de Rennes

\section{Édition imprimée}

Date de publication : 20 décembre 2014

Pagination : 43-54

ISBN : 978-2-7535-4002-6

ISSN : 1956-3485

Référence électronique

Érick Falardeau, Cindy Pelletier et Daisy Pelletier, «La méthode de la pensée à voix haute pour analyser les difficultés en lecture des élèves de 14 à 17 ans », Éducation et didactique [En ligne], 8-3 | décembre 2014, mis en ligne le 20 décembre 2016, consulté le 17 août 2022. URL : http:// journals.openedition.org/educationdidactique/2022 ; DOI : https://doi.org/10.4000/ educationdidactique.2022 


\title{
LA MÉTHODE DE LA PENSÉE À VOIX HAUTE POUR ANALYSER LES DIFFICULTÉS EN LECTURE DES ÉLÈVES DE 14 À 17 ANS
}

\author{
Érick Falardeau, CRIFPE-université Laval (Qc) \\ Cindy Pelletier, CRIFPE-université Laval (Qc) \\ Daisy Pelletier, CRIFPE-université Laval (Qc)
}

Peu de travaux ont documenté les capacités et les difficultés en lecture des élèves francophones du $2^{\mathrm{e}}$ cycle du secondaire - 14 à 17 ans. Pour étudier ces capacités, nous nous concentrerons dans cet article sur des lectures réelles d'élèves de la fin du secondaire. Après avoir présenté l'outil de production de données que nous avons retenu, la méthode de la pensée à voix haute - Think aloud protocols -, nous décrirons la façon dont se sont déroulés les entretiens, ainsi que les résultats que nous dégageons, notamment les difficultés des élèves à construire le sens global du texte, à l'interpréter, à remédier à leurs bris de compréhension et à apprécier un texte qu'ils ne comprennent pas

Mots-clés : Compréhension en lecture, stratégies de lecture, méthode de la pensée à voix haute, enseignement de la lecture, apprentissage continué de la lecture.

High School Students Reading's Difficulties Analyzed with Think Aloud Protocols

Few studies have documented the ability and reading difficulties of francophone high school's students. To investigate these abilities, we will focus in this article on effective reading practices of teenage students. After presenting the data generation tool that we have used, the Think aloud protocols, we'll describe how the interviews were conducted, and the results we disclaim, in particular the difficulties of students to build the overall meaning of the text, to interpret it, to remedy their failure to understand, and appreciate a text they don't understand

Keywords: Reading comprehension, reading strategies, Think aloud protocols, reading instruction. 


\section{INTRODUCTION}

Peu de travaux dans l'univers francophone ont documenté les capacités en lecture des élèves de la fin du secondaire - 14 à 17 ans -, et les composantes $\mathrm{du}$ processus de lecture qui leur posent encore des problèmes. Si des études américaines montrent que la maitrise ${ }^{1}$ des stratégies de lecture est au cœur des problèmes de lecture des élèves plus âgés (Conley, 2009), on en sait encore peu sur la façon dont les adolescents francophones régulent leur activité de compréhension et recourent aux stratégies de lecture.

Pour étudier ces deux composantes de l'activité de lecture - la régulation de l'activité et le recours aux stratégies -, nous nous concentrerons dans cet article sur des lectures réelles d'élèves de la fin du secondaire dans des classes régulières ${ }^{2}$. Nous montrerons comment nous étudions leurs capacités en lecture et leur maitrise des stratégies, afin de mieux accompagner leurs enseignants de français dans un programme de formation continue portant sur l'enseignement des stratégies de lecture. Après avoir présenté l'outil de production de données que nous avons retenu, la méthode de la pensée à voix haute - Think aloud protocols -, nous décrirons la façon dont se sont déroulés les entretiens, ainsi que les résultats préliminaires que nous dégageons après avoir rencontré 43 élèves en septembre 2012, au moment de commencer une formation continue en lecture de 27 heures avec leurs enseignants $(n=12)$.

\section{PRODUIRE DES DONNÉES SUR LES CAPACITÉS EN LECTURE DES ÉLÈVES DE 14-17 ANS}

Les travaux francophones en didactique du français et en psychopédagogie qui ont étudié les processus de lecture des élèves et leurs difficultés se sont surtout concentrés sur les jeunes lecteurs. Les travaux de Giasson (1995, 2000, 2003), qui sont largement diffusés au Québec et en Europe francophone, ne débordent pas du primaire - jusqu'à douze ans. L'ouvrage publié par l'Observatoire national de la lecture (Fayol, 2000) a lui aussi visé les jeunes lecteurs, étudiant par exemple les processus mis en œuvre vers la fin du primaire et au début du secondaire. Les nombreux écrits de Goigoux (2002, 2003 ; Goigoux \& Cèbe, 2004) sur les difficultés des élèves et sur les stratégies d'enseignement efficaces de la lecture concernent surtout les premiers apprentissages de l'écrit, jusqu'à la fin du primaire. Goigoux (2000) a néanmoins réalisé une recherche d'importance en Segpa, avec des élèves en grande difficulté d'apprentissage issus à $97 \%$ de milieux défavorisés. Si le profil des élèves de Segpa ne correspond pas à celui de l'échantillon que nous étudions, leurs capacités en lecture recoupent néanmoins celles que nous avons analysées et que nous présentons dans cet article.

Aux États-Unis, dans le Handbook on Reading Comprehension (Israel \& Duffy, 2009), WartonMcDonald et Swiger (2009) ainsi que Conley (2009) affirment que les études sur les capacités en compréhension des jeunes adultes sont beaucoup moins nombreuses que celles portant sur les lecteurs entrant dans l'écrit, même si en 2007 Cassidy et Cassidy ont listé les études sur la littératie adolescente comme " the hottest topic in litteracy education " (Warton-Macdonald \& Swiger, 2009 , p. 510). Ces articles rétrospectifs pointent eux aussi les stratégies de compréhension comme l'un des domaines à prioriser pour améliorer les capacités en lecture des adolescents, en classe de langue première comme dans les autres disciplines où les élèves lisent des textes de plus en plus difficiles: "Comprehension - getting meaning from text is the purpose of reading, a purpose that becomes increasingly important in the upper grades as students are required to gain increasing amounts of information from more complex texts. » (Hammond Stoughton, 2008, p. 95) Cette complexité accrue s'explique notamment parce que les informations sont davantage imbriquées dans les disciplines scolaires que les élèves doivent maitriser (Buehl, 2007). Pour d'autres chercheurs, le défi de l'apprentissage de la lecture serait plus important au secondaire: «Making fluent readers out of struggling adolescent readers takes about five times the effort that it takes to teach a beginning reader to read, but it can be done. » (Carnine \& Grossen, 2008, p. 200) On sait par ailleurs que les lecteurs compétents recourent à plusieurs stratégies métacognitives pour contrôler leur lecture, réajuster leurs prédictions en fonction de ce qu'ils comprennent du texte; les adolescents en difficulté de lecture se contentent, eux, d'une cohérence minimale et activent très peu leurs processus métacognitifs (Garbe, Holle, Weinhold, Meyer-Hamme, \& Barton, 2010 ; Goigoux, 2000). 


\section{LA MÉTHODE DE LA PENSÉE À VOIX HAUTE}

Pour étudier les capacités en lecture des adolescents, nous recourons aux Think aloud protocols ou Verbal protocols, expressions traduites en français sous la forme de "méthode de la pensée à voix haute » (désormais MPVH). Plusieurs chercheurs ont balisé l'usage de la MPVH en lecture (Gufoni, 1996 ; Hilden \& Pressley, 2011 ; Pressley \& Afflerbach, 1995). Elle consiste à demander à un participant d'exprimer à voix haute tout ce à quoi il est en train de penser pendant qu'il exécute une tâche de lecture, pour rendre « observables » les différents mécanismes de la pensée utilisés en lisant (Gufoni, 1996). Selon Hilden et Pressley (2011), l'utilisation de cette méthode « can enlighten our understanding of such factors as reader characteristics - processes and strategies used by readers, reader's motivation and effect, the interaction of readers' motivation and affect with their cognitive responses - and the examination of contextual variables: text task, setting, and reliability» (p. 431).

Le chercheur doit expliquer aux participants ce qu'il attend d'eux en mentionnant ce que veut dire " penser à voix haute » ou « verbaliser » et jusqu'où ils doivent aller dans le détail et dans la réflexion. Par exemple : "Par "penser à voix haute", nous voulons dire que tu exprimes à voix haute tout ce que tu te dis dans ta tête pendant que tu lis, tout ce à quoi tu penses, ce à quoi tu réagis - rire, surprise, désaccord -, ce que tu comprends, ce que tu ne comprends pas et tout ce que tu fais pour essayer de comprendre le texte. » Pour relancer le participant qui cesse de verbaliser, le chercheur doit utiliser des formules simples qui n'influenceront pas l'activité cognitive du participant : par exemple, " continue à parler ", " continue de penser à voix haute », "n'oublie pas de me dire tout ce qui te passe par la tête », etc. Selon les auteurs consultés, le type de verbalisation retenu sollicite peu de processus supplémentaires à ceux mis habituellement en ouvre et interfèrerait donc peu avec les processus de lecture spontanés du participant (Greene, Robertson, \& Crocker Costa, 2011) ; en outre, il n'accroit presque pas le temps de réalisation de cette tâche (Bowles, 2010).

Dans le cas de notre étude, nous avons respecté ces balises en demandant dans un premier temps à 43 élèves $^{3}$ de lire la nouvelle Solidarité d'Italo Calvino ${ }^{4}$. Ces élèves ont été choisis par leur enseignant; chacun des 12 participants à notre rechercheformation devait identifier dès la rentrée de septembre deux élèves bons lecteurs et deux autres éprouvant des difficultés, dans des classes de $3^{\mathrm{e}}, 4^{\mathrm{e}}$ et $5^{\mathrm{e}}$ secondaire du secteur régulier. Nous souhaitions ainsi obtenir un échantillon varié, dans lequel nous étions susceptibles de rencontrer différents types de lecteurs, mobilisant différentes stratégies. Nous n'avons toutefois pas retenu ces étiquettes pour notre analyse, afin de porter un regard neuf sur leurs capacités, non influencé par le jugement de leur enseignant - qui les connaissait d'ailleurs très peu au moment de les choisir, deux semaines après la rentrée. Cet échantillon a été jugé suffisant pour atteindre un niveau de saturation dans l'identification des capacités en lecture des élèves de 14 à 17 ans.

Une fois la lecture verbalisée complétée, nous avons entrepris une deuxième phase de l'entrevue, au cours de laquelle les intervieweurs ont interrogé les élèves sur leur lecture pour mieux comprendre comment ils détectaient leurs bris de compréhension et recouraient à des stratégies de lecture pour les réparer. Nous nous sommes alors basés sur une liste de questions construite à partir de notre analyse de la nouvelle :

- Qu'est-ce que tu as compris de l'histoire? Qu'as-tu trouvé difficile en lisant?

- Qui sont les personnages ? Que font-ils ? Quels indices du texte te permettent de le savoir? Qui est le narrateur? Quel rôle jouet-il ? Quels indices t'aident à le savoir ?

- Pourquoi le narrateur recommence-t-il à se promener seul au hasard à la fin ?

- Pourquoi l'auteur a-t-il choisi Solidarité pour le titre de sa nouvelle?

- Est-ce que l'histoire t’a fait rire? As-tu repéré de l'humour?

Tous les élèves étant confrontés à une grande incompréhension devant les déplacements et l'identité du narrateur, nous avons voulu avec ces questions vérifier s'ils savaient récupérer de nouveaux indices, trouvés avec l'intervieweur, pour relancer leur travail de compréhension. C'est dans ce travail de résolution de problème que nous avons tenté de déceler l'utilisation de stratégies de lecture.

Cette méthode de questionnement ne permet pas de développer la maitrise des stratégies de lecture des élèves, mais ce n'est pas l'objectif poursuivi. Le schéma d'entretien construit avec la MPVH n'est pas un outil d'enseignement - même s'il pourrait être utilisé comme tel (Israel \& Massey, 2005) - mais un outil de recherche, qui vise d'abord à étudier de quelle 


\section{Érick Falardeau, Cindy Pelletier \& Daisy Pelletier}

façon un élève détecte ses bris de compréhension et tente de les réparer. C'est pour cette raison que nous avons choisi un texte difficile, pour être certains que les élèves éprouvent des problèmes de compréhension, afin de voir de quelle façon ils tenteraient ou non d'y remédier. Notre choix d'un texte difficile se justifie aussi dans la mesure où la lecture individuelle du texte par les élèves est suivie d'un entretien semidirigé au cours duquel un lecteur expert aide les élèves à cheminer dans leur compréhension.

Contrairement à ce qu'en disent plusieurs auteurs cités, dans la MPVH, la verbalisation interfère avec le cours de la lecture ; plusieurs élèves ont affirmé qu'ils avaient trouvé difficile la verbalisation de leurs pensées $^{5}$. La MPVH, employée seule, peut donc empêcher certains élèves de construire le sens global $\mathrm{du}$ récit. Nous avons dans les faits recueilli assez peu de données dans la première moitié des entretiens - lecture individuelle -, les élèves verbalisant peu. Ce sont surtout leurs réponses à nos questions ciblées dans la deuxième phase qui nous ont permis d'explorer leur processus de lecture : les questions qu'ils se posent, les moyens qu'ils se donnent pour détecter et régler leurs problèmes de compréhension. Nous nous sommes donc gardés d'étiqueter comme faibles les élèves qui avaient peu verbalisé, étudiant plutôt dans la deuxième phase de l'entretien leur capacité à remettre en cause leur première lecture et à utiliser des informations pour réfléchir sur un texte. Ainsi, si tous les élèves ont éprouvé des difficultés importantes au cours de leur lecture individuelle, le portrait s'est fortement nuancé à partir des échanges. Certes, ce guidage ne nous permet pas de savoir si les élèves pourraient d'eux-mêmes comprendre un autre texte, il nous permet néanmoins de vérifier s'ils savent utiliser des indices relevés dans Solidarité pour essayer de réparer leurs bris de compréhension.

\section{Les outils d'analyse}

Pour effectuer l'analyse qualitative des entretiens réalisés, nous avons élaboré une grille de catégorisation basée sur un cadre théorique dans lequel le processus de lecture se décline en un certain nombre de composantes itératives (Giasson, 1995 ; Cèbe, Goigoux, \& Thomazet, 2007 ; Falardeau, Guay, \& Valois, 2012) que nous avons regroupées en six catégories : planification, compréhension, interprétation, réaction, mobilisation de connaissances, métacognition.
La composante « Compréhension » est la plus sollicitée; nous l'avons découpée en six sous-composantes pour l'appréhender plus finement :

- microprocessus - verbalisation ou manifestation de l'utilisation de stratégies liées à la compréhension des mots et des syntagmes ;

- macroprocessus - verbalisation ou manifestation de l'utilisation de stratégies liées à la compréhension globale du texte ou d'un segment important ;

- processus d'intégration - verbalisation ou manifestation de l'utilisation de stratégies liées à la compréhension du lien entre les énoncés (reprises, marqueurs de relation, organisateurs textuels, reconstruction de la chaine logique ou temporelle entre 2-3 énoncés sans résumer l'histoire);

- visualisation du contenu - verbalisation par l'élève des images qu'il se construit mentalement pendant la lecture du récit.

L'interprétation recoupe les catégories liées à l'intention de l'auteur, au titre, à la psychologie et au comportement des personnages, à la portée symbolique de l'intrigue. Une autre catégorie particulièrement sollicitée lors de nos entretiens, la métacognition, comprend les catégories suivantes :

- réflexion par rapport à l'expérience générale de lecture ou à l'utilisation générale des stratégies - sans lien avec le texte lu ;

- détection des pertes de compréhension - l'élève se rend compte qu'il ne comprend plus (ou pas), mais ne précise pas la nature de son incompréhension ; nous avons subdivisé cette catégorie pour isoler les pertes de compréhension par rapport aux mots, aux liens entre les phrases ou les idées, et par rapport au fil de l'histoire, l'intrigue ;

- réparation des pertes de compréhension - les moyens pris par l'élève pour réparer des pertes de compréhension (relecture, inférences, etc.) au moment où il le fait ;

- réflexion par rapport à l'expérience de lecture ou à l'utilisation des stratégies dans le texte lu - Comment a-t-il vécu sa lecture ? Quelle stratégie a-t-il utilisée ? Comment l'at-il utilisée ? Pourquoi ? Qu'en a-t-il retiré ?

Chaque entretien d'une durée approximative de 25 minutes a été codé à l'aide de ces catégories, les membres de notre équipe ayant procédé à une validation intercodeurs tous les cinq entretiens traités pour s'assurer qu'ils partageaient une compréhension commune des codes. 


\section{ANALYSES DE RÉSULTATS}

\section{Centration sur les processus d'intégration et sur les microprocessus au détriment des macroprocessus}

Pendant la lecture individuelle, les élèves reformulaient des passages ou liaient les phrases entre elles (processus d'intégration), mais seulement 14 élèves sur les 43 (33\%) ont cherché à replacer les segments dans le déroulement de l'intrigue, en activant des macroprocessus.

À titre d'exemple, Mathieu, dans la première partie de l'entretien, tente régulièrement de construire du sens en reformulant des portions du texte. Toutefois, il comprend les actions des personnages de manière morcelée, les paraphrasant au fur et à mesure qu'il les lit, sans les intégrer à un tout cohérent pour comprendre l'intrigue du récit :

é : On dirait qu'ils veulent aller voler de quoi, je trouve.

$[\ldots]$

é : Dans le fond, on dirait qu'ils rentrent, ils sont cachés, le dos accoté...

$[\ldots]$

é: Halte, qui va là ! Il y a quelqu'un qui les surprend.

$[\ldots]$

é : Seul au hasard... Là, à la fin, il est tout seul et il marche... il s'est sauvé.

En dissociant les processus d'intégration et les macroprocessus, les élèves ne récupèrent pas les indices inférés à partir des phénomènes de reprise ou des marqueurs de relation pour construire le sens du récit. Irwin plaide plutôt pour une intégration de ces processus : " Macro - and integrative processes interact in that local connection provide cues as to the global connections, and vice versa » (1986, p. 44-45).

Ces lacunes pourraient être attribuées à la charge cognitive qu'entraîne la verbalisation dans la MPVH, les élèves se limitant à paraphraser le texte au lieu d'en comprendre l'intrigue. Elles peuvent aussi être dues à la nature des problèmes de compréhension que pose la tâche de lecture : les élèves ont éprouvé des difficultés à se centrer sur le sens global de l'histoire parce que le narrateur change constamment de groupe et qu'il est difficile de voir à quel moment il se déplace, de quelle façon il le fait et pour quels motifs, qui sont les personnages avec qui il interagit. C'est cette incompréhension que nous voulions provoquer, en observant de quelle façon les élèves en prenaient conscience et tentaient d'y remédier.

Une autre explication peut être avancée : les élèves, notamment les plus faibles, sont peu conscients que le passage aux macroprocessus est indispensable à la compréhension d'un récit, comme les adolescents interrogés par Goigoux (2000) qui « semblent ignorer la nécessité d'élaborer des représentations provisoires au fur et à mesure de la lecture du texte » (p. 151), pour construire une compréhension d'ensemble. Ces élèves n'ont pas développé une conscience métacognitive qui leur permettrait d'identifier plus clairement la finalité de leur lecture: appréhender une intrigue plutôt que des épisodes morcelés. Au fur et à mesure qu'ils progressent dans leur lecture individuelle, seulement $25 \%$ des élèves font montre d'une forte conscience métacognitive, concentrant leur attention sur les enjeux centraux du texte, notamment l'ambigüité des personnages et les indications confuses par rapport aux déplacements du narrateur entre le groupe des cambrioleurs et celui des policiers.

Nos données soutiennent cette hypothèse quant à la faible conscience métacognitive des élèves en difficulté : ceux-ci n'ont pas été capables de mobiliser les macroprocessus, même avec aide. Les 25 \% d'élèves ayant tiré profit du sens de phrases ou de passages pour essayer de construire le sens global du texte ont surtout illustré leur compétence dans la deuxième partie de l'entrevue, lorsqu'ils étaient guidés par des questions de l'intervieweur. Ce soutien a diminué la complexité de la tâche de lecture, rendant ainsi possible, pour les meilleurs élèves, la mobilisation de macroprocessus.

Dans l'extrait suivant, Bianca, tout en tentant de comprendre le sens d'une action, essaie de la replacer dans l'économie globale du récit :

I : Là, qu'est-ce qui se passe ici ? Lignes $55-56 \ldots$

é : Ben ils l'ont envoyé rentrer voir le magasin.

I : Ok.

é : Le... « Je».

I : Le «Je ». Le narrateur, il est rentré dans le magasin. Donc, physiquement là, si on se fait un film, il est avec les polices, puis il a fait quoi ? 


\section{Érick Falardeau, Cindy Pelletier E Daisy Pelletier}

é : Puis là, il s'en va en dedans pour euh... pour faire l'éclaireur.

I : Continue à lire. Ah, c'est bien ça, faire l'éclaireur ! Continue ici, Désormais...

é : Désormais, disait quelqu'un se mettant un sac sur le dos, ils ne peuvent plus nous attraper ! Là, il est rendu avec les (rire discret)... les bandits !

I : Pourquoi ? Qu'est-ce qui te dit... Pourquoi tu es si sure que ça?

é : Ben parce que sinon il les aurait attrapés.

I : Ok. Donc c'est un voleur qui dit ils ne peuvent plus nous attraper. Est-ce que c'est clair que c'est un voleur?

é : Non. C'est pas clair, mais si on le relit puis qu'on décortique, là, c'est sûr que...

I : C'est facile. Vite! Dis un autre, tirons-nous par l'arrière-boutique, comme ça nous leur filons sous le nez.

é : Nous filons sous le nez de la police.

I : Excellent. Nous avions tous un sourire de triomphe sur les lèvres.

é : Justement, il est devenu avec les voleurs.

I : Il est redevenu... il est devenu avec les voleurs. Ça se peut pas...?

é : Ben il a... il a fait voleurs-polices-voleurs.

L'intervieweur ne lui suggère pas les réponses; il l'amène à observer des passages qui lui ont probablement échappé lors de sa première lecture. La dernière phrase résume l'aboutissement de la réflexion de Bianca sur le sens global de la nouvelle : elle a intégré chacune des actions morcelées du récit en un tout provisoirement cohérent qui lui permet de comprendre que le personnage principal est voleur puis policier.

\section{La mobilisation des macroprocessus pour passer à l'interprétation}

Dans un texte difficile, seuls les élèves qui activent les macroprocessus, qui tentent de construire le sens global du récit et y arrivent avec un certain succès, réussissent à avancer des interprétations quant au sens du titre, aux intentions des personnages, de l'auteur, etc.

I : Je veux juste poser une dernière question... sur le titre. Qu'est-ce que ça veut dire Solidarité? é : Travail d'équipe je pense.

I : Travail d'équipe, aider les autres... quand tu es solidaire, tu aides les autres. Pourquoi Italo Calvino, c'est le nom de l'auteur ça, Italo Calvino, pourquoi il a appelé son histoire Solidarité ?

é : Ben il aide les deux groupes... je pense.

I : Parce qu'il aide les deux groupes...

é : Ben... il aurait pu soit se mettre dans un groupe puis nuire à l'autre ou nuire à l'autre, mais il n'a rien fait pantoute, fait qu'il aide les deux.

$\mathrm{I}$ : Il aide les deux, donc, est-ce que le titre, il marche?

é : Ouais... mais... il est solidaire à personne. Parce que s'il avait été solidaire aux policiers, il les aurait aidés pour attraper les voleurs, puis... les voleurs, il les aurait aidés, mais il n'a rien fait.

I : C'est de la vraie solidarité ?

é : Non, ben je ne pense pas là... mais dans un sens, il ne nuit pas aux deux, fait qu'il est un petit peu solidaire avec les deux.

I : Il ne nuit pas aux deux, mais est-ce qu'il aide les deux?

é : Non.

I : Il les aide un peu, mais pas beaucoup.

é : Il les aide un peu, mais dans le fond, s'il en aide un, ça nuit à l'autre, et s'il en aide l'autre, ça nuit à l'un, fait que ça s'annule je pense. (Camille)

L'élève qui interprète ainsi la signification du titre, en voyant bien que le protagoniste n'est pas solidaire puisqu'il trahit en quelque sorte les deux groupes auxquels il se joint, montre bien qu'elle conçoit qu'un texte puisse être absurde en rompant avec les attentes de cohérence du lecteur. Elle active aussi plusieurs processus de compréhension qui l'aident à percevoir l'absurde : les plus manifestes sont les macroprocessus, parce qu'elle a su lier les épisodes où le protagoniste devenait tour à tour voleur puis policier. Sans ce travail de compréhension et cette ouverture à l'absurdité, l'élève n'aurait pu interpréter le titre comme un paradoxe, un contresens. Cette donnée n'est pas inédite (National Reading Panel, 2000), mais elle réitère la nécessité d'enseigner les stratégies de compréhension et de confronter les élèves à une grande variété de textes littéraires si l'on veut les amener à interpréter des textes aussi complexes que Solidarité qui présentent des caractéristiques artistiques souvent déconcertantes. 
En revanche, un élève qui n'a pas compris comment, au fil du récit, le personnage principal passait indifféremment d'un groupe à un autre peinera à construire une interprétation qui s'appuie de façon pertinente sur le texte :

I : Est-ce que tu penses que l'auteur, c'est pour une raison en particulier qu'il n'utilise pas de pronoms pour désigner ses personnages? Tu as remarqué, dans le texte, c'est beaucoup des ils, des nous... Penses-tu qu'il y a une raison en particulier pour laquelle on fait ça ?

é : Il... d'après moi, il voulait pas trop donner des noms au hasard pour pas que ça tombe sur des vraies personnes.

I : Il ne voulait pas que ce soit de vrais criminels qui soient désignés par des prénoms?

é : Ouin.

I : OK. Fait que ce serait une question d'anonymat, c'est ça que tu me dis?

é : Ouin. (Anthony)

Contrairement à d'autres élèves plus forts, cet élève n'a pas compris l'identité des groupes auxquels se joint le personnage principal ; il éprouve donc des difficultés à interpréter les choix d'écriture de Calvino, qui tait l'identité des voleurs et des policiers en utilisant bon nombre de pronoms ambigus - les autres, ils, nous...

\section{Détection des pertes de compréhension et tentative de remédiation}

Dans la première partie de l'entretien, $37 \%$ des élèves ont mobilisé d'eux-mêmes la stratégie Détection des pertes de compréhension par rapport à l'intrigue; en revanche, tous se sont interrogés à un moment ou à un autre sur la compréhension des mots difficiles ou du lien entre les phrases. Irwin (1986) permet de bien saisir les raisons pour lesquelles les stratégies de détection et de réparation des pertes de compréhension liées à l'intrigue sont peu mobilisées par la plupart des élèves lorsqu'on ne les y invite pas. Plus précisément, elle présente une taxonomie des moyens disponibles pour remédier aux bris de compréhension et classe ces moyens selon cinq niveaux, du moins couteux au plus couteux sur le plan cognitif :
Figure 1 : Moyens disponibles pour remédier aux bris de compréhension (traduction libre)

\begin{tabular}{|c|c|c|}
\hline \multirow[t]{5}{*}{$\begin{array}{c}\text { Moins } \\
\text { couteux }\end{array}$} & 1 & $\begin{array}{l}\text { Ignorer le bris de compréhension et pour- } \\
\text { suivre la lecture }\end{array}$ \\
\hline & 2 & Retarder la formulation d'hypothèses \\
\hline & 3 & Formuler une hypothèse plausible \\
\hline & 4 & $\begin{array}{l}\text { Relire sur le champ la ou les phrases non } \\
\text { comprises }\end{array}$ \\
\hline & 5 & $\begin{array}{l}\text { Relire le contexte qui précède le bris de } \\
\text { compréhension }\end{array}$ \\
\hline Plun & 6 & Consulter une source experte \\
\hline
\end{tabular}

Sans l'aide de l'intervieweur, la majorité des élèves en difficulté recourent à des moyens de premier, deuxième et troisième niveaux, moins coûteux sur le plan cognitif, mais insuffisants pour remédier à la plupart des bris de compréhension qu'ils sont susceptibles de rencontrer au secondaire. Samuel, par exemple, détecte un problème, mais n'interrompt pas sa lecture pour tenter d'y remédier :

é : Je ne comprends plus rien. Il y en avait qui rasaient les murs, cachés dans les renfoncements... Je ne comprends rien. (Samuel)

Peu d'élèves relisent d'eux-mêmes une phrase (niveau 4) (25\%) ou le contexte qui précède le bris de compréhension (niveau 5) (37\%), bien qu'il s'agisse d'un moyen-clé pour résoudre les problèmes que pose le texte Solidarité :

é : J'essaye tout le temps de revenir en arrière parce que... ils disent... on dirait que là c'est les bandits qui parlent puis là c'est ils ont attrapé quelqu'un, fait que j'imagine que c'est la police qui a attrapé quelqu'un. (Bianca)

Dans cet extrait, Bianca reconnait d'elle-même être obligée de relire régulièrement certains passages parce qu'elle ne sait plus si ce sont les voleurs ou les policiers qui parlent.

D'autres élèves donnent quant à eux l'impression de ne pas savoir s'ils ont compris ou non l'histoire. Ils attendent donc des questions susceptibles d'évaluer leur compréhension :

é : Il faut que je relise l'histoire? 


\section{Érick Falardeau, Cindy Pelletier E Daisy Pelletier}

$\mathrm{I}:$ Je ne sais pas, qu'est-ce que tu fais?

é : Euh... habituellement, il y a des questions, puis avec les questions, je réponds...

I : Mais là, il n'y a pas de questions. Ce n'est pas un exercice dans une classe là ! (Mathieu)

Cet extrait illustre bien la dépendance de certains élèves au questionnaire qui suit traditionnellement la lecture d'un texte scolaire, ce que Cèbe, Goigoux et Thomazet (2007) confirment : «Quand on leur demande: "comment je peux savoir si tu as compris ce texte", ils répondent "il faut me poser des questions" » (p. 3). C'est ainsi au terme de leur lecture individuelle, lorsque l'intervieweur leur demande de résumer l'histoire, que la majorité des élèves de notre échantillon réalisent qu'ils n'ont pas saisi l'intrigue de l'histoire : ils ont compris des épisodes, pris isolément, mais n'ont pas compris l'histoire.

Globalement, les élèves qui détectent leurs bris de compréhension pendant la première partie de l'entretien ont du mal à réparer ces pertes, soit parce qu'ils sont en surcharge cognitive, soit parce qu'ils ne savent pas quel moyen mettre en œuvre, contrairement aux bons lecteurs, qu'Irwin (1986) décrit comme plus habiles sur le plan métacognitif : "Good readers know when they have or have not understood something, and they know what to do when their comprehension breaks down " (p. 86). Quant aux élèves qui n'ont pas ou qui ont peu conscience de leurs bris de compréhension, ils sont évidemment peu portés à mobiliser des stratégies de remédiation au cours de leur lecture.

\section{Satisfaction des élèves qui réussissent à comprendre}

Les élèves qui, au terme de l'entretien, ont l'impression d'avoir compris l'histoire expriment beaucoup de satisfaction. Ils ont résolu un problème complexe intellectuellement motivant :

(À 13 minutes 35, au terme de la première lecture sans accompagnement)

é : Sincèrement, je trouve que ça n'a pas de but vraiment !

I : Tu trouves que l'histoire n'a pas de but?

é : Pas vraiment, je ne comprends pas trop.

(À 15 minutes 10)
I : «Quand on dit Puis, ils me dirent d'aller voir dehors, jusqu'au coin, s’il n'arrivait personne. Le ils ici, ce serait qui ?

é : Euh, la gang de voleurs.

I : La gang de voleurs qui auraient dit ça... ?

é : Au personnage principal.

I : Au personnage principal. J'y allai. Au coin de la rue, dehors, il y en avait qui rasaient les murs, cachés dans les renfoncements et qui s'avançaient. Je m'y mis moi aussi.

é : Ah ! [intonation de fierté]

I : Qu'est-ce que ça veut dire ça ?

é : Il s'est mis avec eux.

I : Qui s'est mis avec qui ?

É : Ok ! Là, je comprends mieux ! C'est le personnage principal qui s'est mis avec les autres qui étaient dans la rue!

(À 26 minutes 30, à la fin de l'entretien)

I : C'est une histoire que tu as quand même aimée?

é : Ben... c'est quand même une bonne histoire, mais elle est compliquée.

I : Pourquoi c'est une histoire que tu aimes maintenant que tu la comprends?

é : Ben... c'est que... comment je pourrais dire...

I : Qu'est-ce qu'il y a de fun dans cette histoire-là ? Je te vois sourire...

é : J'aime ça quand c'est complexe puis que je comprends.

I : Il y a une satisfaction là-dedans ?

é : Ouais. (Olivier)

La progression de l'entretien dans la deuxième partie, quand l'intervieweur pointe à l'élève des extraits complexes comme ceux où le protagoniste passe d'un groupe à l'autre, montre de quelle façon le sentiment de réussite est associé à la compréhension du récit. C'est le sentiment d'avoir réussi une tâche complexe qui nourrit la motivation. C'est à cette condition que l'histoire peut devenir drôle, lorsque les élèves comprennent.

Pour leur part, les élèves qui avouent ne rien comprendre à cette histoire disent ne pas trop l'avoir aimée, soulignant eux aussi à leur manière l'imbrication macroprocessus/réaction :

I : Est-ce que c'est une histoire que tu as aimée? 
é : Pas full là... je ne comprenais pas full puis...

I : À cause de ça tu penses?

é : Ouin... je ne savais pas c'était quoi le but puis qu'est-ce [que le personnage principal] voulait. (Érika)

Érika lie son manque de motivation à sa mauvaise compréhension du texte. Or, les textes littéraires et courants donnés à lire aux élèves à la fin du secondaire sont souvent d'une grande complexité. Les textes difficiles par leur langage, leur caractère absurde, leur construction narrative ou leur propos doivent être travaillés avec les élèves parce qu'ils posent des problèmes dont la résolution constitue une occasion d'apprentissage. L'enseignant doit utiliser ces problèmes pour enseigner des stratégies de lecture, que les élèves apprendront à mobiliser avec de plus en plus d'autonomie, en étant de moins en moins accompagnés, pour qu'ils développent le gout de lire, même à 15 ou 17 ans.

\section{CONCLUSION}

Une telle étude présente des limites méthodologiques : la MPVH, malgré toutes les précautions prises, interfère avec les processus normaux de lecture. En se concentrant pour verbaliser ce qui se passe dans leur tête au moment où ils lisent, les élèves ajoutent à la charge cognitive engendrée par la complexité du texte à lire, quoi qu'en disent les articles à caractère méthodologique qui ont analysé la mise en œuvre des Think Aloud Protocols. La tâche de verbalisation n'est pas sans poser problème :

I : Est-ce qu'il se passe des choses dans ta tête... que tu ne me dis pas?

é : Euh... Ben je ne sais pas vraiment là...

I : Qu'est-ce que tu ne sais pas?

é : Non, ben... quoi... quoi dire, puis tout là... (Érika)

I : C'est dur comme texte, hein?

é : C'est plus dur décrire ce qui se passe dans ma tête. (Anthony)

Malgré ces difficultés inhérentes à toute cueillette de données auprès d'élèves, l'étude que nous menons nous permet de dégager des pistes pour l'enseignement continué de la lecture auprès d'adolescents de la fin du secondaire. Livrés à eux-mêmes devant un texte complexe comme Solidarité, les deux-tiers (29/43) des élèves rencontrés concentrent leur attention sur la compréhension des mots, des syntagmes et de courts segments de textes. C'est là une lacune importante pour la réussite de leurs études, car dans les programmes par compétences, les élèves lisent des textes complexes non seulement en français, mais aussi en histoire, en sciences, en mathématiques. Leurs faibles capacités à reconstruire le propos d'un texte constituent ainsi un frein à leur réussite dans toutes ces disciplines.

Mais la deuxième partie de nos entretiens permet d'entrevoir des pistes de solution, les élèves remettant en question leur première lecture en entrant en dialogue avec un lecteur plus compétent, même à 15,16 ou 17 ans : "The philosophy that it is too late to make a difference by the time a student reaches the upper grades and that all that we can do is simply help them "get through" the assigned work, is no longer viable. " (Hammond Stoughton, 2008, p. 89) Aucun des six élèves jugés forts lecteurs (14\%) - ceux qui parviennent à démêler les fils de l'intrigue au terme de l'entretien - ne réussit seul à comprendre que le récit présente un seul narrateur jouant à la fois au policier et au voleur, et ce, par hasard. Toutefois, leur conscience métacognitive leur permet de recoller les morceaux du casse-tête pour construire une intrigue cohérente; ils sont attentifs aux passages que leur pointe l'intervieweur, ce qui leur permet à la fin de s'interroger sur le titre et sa signification, interprétation à laquelle ne peuvent se livrer les élèves qui ne comprennent pas le comportement absurde du narrateur. En créant des situations didactiques où les élèves se retrouvent devant un texte résistant, l'enseignant les entraine à s'ouvrir à la diversité des œuvres d'art dont le propos ou la construction remettent souvent en question les attentes de cohérence du lecteur, ses représentations de ce que doit être un récit, une intrigue, etc. Il leur enseigne aussi à s'approprier des stratégies opérationnelles, dont ils apprendront à questionner la pertinence grâce à l'accompagnement, la discussion. Certains dispositifs didactiques, comme la Démarche stratégique d'enseignement de la lecture mise au point et expérimentée par J. Lecavalier et S. Richard (2011), ou les cercles de lecture entre pairs (Hébert, 2012) montrent toute l'importance de l'intersubjectivité dans l'apprentissage de la compréhension et de l'interprétation de textes complexes. Néanmoins, ces dispositifs ne constituent pas une panacée : des élèves invités à 


\section{Érick Falardeau, Cindy Pelletier E Daisy Pelletier}

discuter de leur compréhension n'apprendront pas à utiliser des stratégies de lecture par imprégnation ; l'enseignement explicite des stratégies, à travers le modelage et la pratique guidée collaborative, a été maintes fois reconnu dans des méta-analyses comme une méthode d'enseignement efficace, particulièrement auprès des lecteurs en difficulté (Gersten, Fuchs, Williams, \& Baker, 2001).

Ces données tirées de méta-analyses américaines méritent toutefois d'être lues et transposées avec prudence. Les recherches en lecture que nous menons avec des enseignants du secondaire depuis 2009 nous ont montré que l'enseignement des stratégies de lecture est loin d'être répandu ; les stratégies sont mentionnées dans les manuels, mais elles font peu l'objet d'un enseignement explicite, accompagné. Or, c'est justement pour tenter de pallier ces manques dans le système d'éducation québécois que nous avons entrepris la présente recherche-formation : en accompagnant des enseignants pendant cinq journées de formation, en explorant avec eux la façon dont ils peuvent utiliser leur matériel didactique pour enseigner les stratégies de lecture de façon explicite, nous souhaitons qu'ils s'approprient des pratiques pédagogiques efficaces, dans les meilleures conditions de formation continue possibles. Selon Desimone (2009), un programme de formation professionnel sera efficace pour modifier les pratiques pédagogiques des enseignants et par le fait même favoriser la réussite scolaire des élèves, s'il met l'accent sur les cinq éléments suivants : 1- Le contenu de la formation permet une meilleure connaissance des pratiques pédagogiques jugées efficientes pour la réussite scolaire des élèves. 2- Les enseignants observent des pratiques pédagogiques réelles au moyen de la vidéo, qu'eux-mêmes ou leurs pairs mettent en ouvre. 3- La formation est en lien avec les programmes et les pratiques les plus courantes. 4- Le temps consacré à la formation jugé optimal est d'une vingtaine d'heures. 5- Plusieurs enseignants d'une même école participent au programme de formation afin d'échanger pour enrichir leur pratique professionnelle. Ces cinq ingrédients clés ont été intégrés à notre programme de formation continue. Et les données analysées dans cet article s'inscrivent dans cette démarche de formation; elles visent à documenter au mieux les capacités des élèves des enseignants participants, afin d'aider ces derniers à comprendre les difficultés de leurs élèves, pour améliorer leurs pratiques d'enseignement de la lecture.

\section{NOTES}

1. Ce texte adopte l'orthographe rectifiée.

2. Il ne s'agit ni d'élèves inscrits dans des programmes enrichis ni d'élèves en grande difficulté qui seraient relégués dans les classes spéciales.

3. Certains élèves n'ont pas été considérés lors de l'analyse parce qu'ils ont retiré leur consentement.

4. Cette nouvelle de quatre pages présente des problèmes de lecture importants qui nous ont permis d'étudier la façon dont les élèves prennent conscience de leurs problèmes de compréhension et les stratégies qu'ils se donnent pour y remédier : un narrateur anonyme passe au hasard une nuit devant une boutique que cambriole un groupe de malfaiteurs. Il se joint spontanément à eux et les aide à perpétrer leur vol. Après quelques minutes, il sort vérifier si des policiers arrivent et se joint à eux, toujours par hasard, pour attraper les voleurs. Il pénètre ensuite dans la boutique, rejoint les voleurs pour les aider, puis redevient policier lorsque ces derniers rattrapent les voleurs. À la fin de l'histoire, après une course folle où il devient tour à tour voleur et policier, le narrateur se fait semer par les deux groupes et recommence à se promener seul, au hasard. Ce récit présente des problèmes de compréhension importants en raison de l'identité floue du personnage principal et des protagonistes de l'histoire; l'action est absurde (un personnage principal qui devient tour à tour voleur et policier) et les déplacements sont difficiles à saisir. Le vocabulaire et la syntaxe sont néanmoins très simples.

5. La MVPH reste malgré cette réserve une méthode de recherche hautement recommandée par les auteurs que nous avons cités. Lorsqu'il s'agit d'avoir accès à des stratégies « invisibles » (Afflerbach \& Cho, 2008, p. 72), aucune méthode n'est parfaite; la méthode permet au mieux d'émettre des inférences ou des hypothèses au sujet de l'utilisation des stratégies et de leur développement. D'autres méthodes présentent par exemple le désavantage de suggérer aux élèves des stratégies potentielles (Goigoux, 2000), ce qui à notre avis interfère encore plus que la MPVH avec le processus de lecture normal des élèves. 
Érick Falardeau, Cindy Pelletier \& Daisy Pelletier

\section{RÉFÉRENCES}

Afflerbach, P. \& Cho, B.-Y. (2008). Identifying and describing constructively responsive comprehension strategies in new and traditionnal forms of reading. In S. Israel \& G. Duffy (Eds.), Handbook of reading comprehension research (pp. 69-90). Mahwah, NJ: Lawrence Erlbaum Associates.

Bowles, M. (2010), The Think-Aloud Controversy in Second Language Research, New York: Routledge.

Buehl, D. (2007). A Professionnal Development Framework for Embedding Comprehension Instruction Into Content Classrooms. In J. Lewis \& G. Moorman (Eds.), Adolescent Literacy Instruction. Policies and Promising Practices (pp. 192-211). Newark, Del.: International Reading Association.

Carnine, L. \& Grossen, B. (2008). Action Planning for Struggling Students to Improve Literacy and Content Area Achievement. In L. Denti \& G. Guerin (Eds.), Effective Practice for Adolescents with Reading and Literacy Challenges (pp. 195-222). New York: Routeledge.

Cassidy, J. \& Cassidy, D. (2007). What's hot, What's not for 2007 ? Reading Today, 24(4), 1-5.

Cèbe, S., Goigoux, R. \& Thomazet, S. (2007). Enseigner la compréhension: principes didactiques, exemples de tâches et d'activités. Disponible en ligne : http://www.ac-caen. fr/ia50/circo/stlol/spip.php?articlell. Consulté le 14 avril 2014.

Conley, M.W. (2009). Improving Adolescent Comprehension. Developing Comprehension Strategies in the Content Areas. In S. Israel \& G. Duffy (Eds.), Handbook on Reading Comprehension (pp. 531-550). New York: Routeledge.

Desimone, L. M. (2009). Improving Impact Studies of Teachers' Professional Development: Toward Better Conceptualizations and Measures. Educationnal Researcher, 38(3), 181-199. Disponible en ligne: http://edr.sagepub.com/content/38/3/181. Consulté le 14 avril 2014

Ericsson, K.A. \& Simon, H.A. (1984), Protocol Analysis: verbal reports as data, Cambridge, MA: MIT Press.

Falardeau, É. \& Gagné, J.-C. (2012). L'enseignement explicite des stratégies de lecture : des pratiques fondées par la recherche. Enjeux, 83, 91-118.

Falardeau, É., Guay, F. \& Valois, P. (2012). Lévaluation de la lecture au secondaire : la transformation du curriculum prescrit en outils d'évaluation valides. In J.-L. Dumortier, J. Van Beveren \& D. Vrydaghs (Éds.), Curriculum et progression en français. Actes du $11^{\mathrm{e}}$ colloque de l'AIRDF (p. 589-610). Namur: Diptyque.

Fayol, M. (2000) (Éd), Maitriser la lecture : poursuivre l'apprentissage de la lecture de 8 à 11 ans, Paris: Odile Jacob.

Garbe, C., Holle, K. \& Weinhold, S. (2010). The Most Important Findings of ADORE and Their Consequences. In C. Garbe, K. Holle, \& S. Weinhold (Eds.), ADORE - Teaching Struggling Adolescent Readers in European Countries (pp. 225-238). Frankfurt: Peter Lang.
Gersten, R., Fuchs, L.S., Williams J.P. \& Baker S. (2001). Teaching Reading Comprehension Strategies to Students With Learning Disabilities: A Review of Research. Review of Educational Research, 71(2), 279320.

Giasson, J. (2003), La lecture : de la théorie à la pratique, Boucherville : Gaëtan Morin Éditeur.

Giasson, J. (2000), Les textes littéraires à l'école, Boucherville : Gaëtan Morin Éditeur.

Giasson, J. (1995), La compréhension en lecture, Boucherville : Gaëtan Morin Éditeur.

Goigoux, R. \& Cèbe, S. (2004). Favoriser le développement de compétences phonologiques pour tous les élèves en grande section de maternelle. Repères, 28, 45-53.

Goigoux, R. (2003). Enseigner la compréhension : l'importance de l'auto-régulation. In M. Fayol \& D. Gaonac'h (Éds.), Aider les élèves à comprendre: du texte au multimédia (p. 182-204). Paris: Hachette.

Goigoux, R. (2002). Analyser l'activité d'enseignement de la lecture : une monographie. Revue française de pédagogie, 138, 125-134.

Goigoux, R. (2000), Les élèves en grande difficulté de lecture et les enseignements adaptés, Suresnes : Éditions du CNEFEI.

Greene, J., Robertson, J. \& Crocker Costa, L.-J. (2011). Assesssing Self-Regulated Learning Using Think-Aloud Methods. In B. Zimmerman \& D. Scunck (Eds.), Handbook of Self-Regulation of Learning and Performance (pp. 313-322). New York: Routledge.

Gufoni, V. (1996). Les protocoles verbaux comme méthode d'étude de la production écrite : approche critique. Études de linguistique appliquée, 101(1), 20-32.

Hammond Stoughton, E. (2008). "I am Never Going to Be a Reader": Issues of Literacy in the Upper Grades. In L., Denti \& G., Guerin (Eds.), Effective Practice for Adolescents with Reading and Literacy Challenges (pp. 85-105). New York: Routeledge.

Hébert, M. (2012). Loral réflexif dans les cercles littéraires entre pairs : vers quels indices de progression ? In J.-L., Dumortier, J., Van Beveren \& D. Vrydaghs (Éds.), Curriculum et progression en français. Actes du $11^{\mathrm{e}} \mathrm{col}-$ loque de l'AIRDF (p. 335-362). Namur: Diptyque.

Hiden, K. \& Pressley, M. (2011). Verbal Protocols of Reading. In N. K. Duke \& M. H. Mallette (Eds.), Literacy Research Methodologies (pp. 427-440). New York : Guilford Press.

Irwin, J. (1986). Teaching Reading Comprehension Processes, Englewood, NJ: Prentice-Hall.

Israel, S. \& Duffy, G. (Eds.), Handbook on Reading Comprehension, New York: Routeledge.

Israel, S. E. \& Massey, D. (2005). Metacognitive ThinkAlouds: Using a Gradual Release Model With Middle School Students. In S. E. Israel, C. Collins Block, K. L. Bauserman \& K. Kinnucan-Welsch (Eds.), Metacognition in Literacy Learning. Theory, Assessment, Instruction, and Professionnal Development (pp. 183-198). Mahwah, NJ: Lawrence Erlbaum Associates. 


\section{Érick Falardeau, Cindy Pelletier E Daisy Pelletier}

Lecavalier, J. \& Richard, S. (2011). Deux stratégies de compréhension et d'interprétation de la langue littéraire. In S. Aeby Daghé (Éd), Enseigner la langue et la littérature. Des dispositifs pour penser leur articulation (p. 137-155). Collection Diptyque. Namur : Presses universitaires de Namur.

National Reading Panel (2000). "Teaching Children To Read" : An Evidence-Based Assessment of the Scientific Research Literature on Reading and Its Implications for Reading Instruction (Rapport de recherche, U.S. Department of Health and Human Services). Disponible en ligne : https://www.nichd.nih.gov/publications/pubs/ nrp/Pages/smallbook.aspx.. Consulté le 14 avril 2014.

Pressley, M. \& Afflerbach, P. (1995), Verbal Protocols of Reading: The Nature of Constructively Responsive Reading, Hillsdale, NJ: Lawrence Erlbaum Associates

Wharton-McDonald, R. \& Swiger, S. (2009). Developing Higher Order Comprehension in the Middle Grades. In S. Israel \& G. Duffy (Eds.), Handbook on Reading Comprehension (pp. 510-530). New York : Routeledge. 